\title{
On generalized derivations in Banach algebras
}

\author{
by \\ NAdia Boudi and SAID Ouchrif (Zitoune Meknes)
}

\begin{abstract}
We study generalized derivations $G$ defined on a complex Banach algebra $A$ such that the spectrum $\sigma(G x)$ is finite for all $x \in A$. In particular, we show that if $A$ is unital and semisimple, then $G$ is inner and implemented by elements of the socle of $A$.
\end{abstract}

1. Introduction. The notion of generalized derivation is due to Brešar [6]. Let $A$ be an algebra. A linear mapping $G: A \rightarrow A$ is called a generalized derivation if there exists a derivation $d: A \rightarrow A$ such that $G(x y)=(G x) y+x d y$ for all $x, y \in A$. A linear map $T: A \rightarrow A$ is called a left centralizer in case $T(x y)=(T x) y$ for all $x, y \in A$. If $G$ is a generalized derivation determined by a derivation $d$, then $G-d$ is a left centralizer. Hence a generalized derivation is a sum of a derivation and a left centralizer. We say $G$ is inner if there exist $a, b \in A$ such that $G x=a x+x b$ for all $x \in A$. Obviously, if the algebra is unital then $G x=(G 1) x+d x$ for all $x \in A$; in this case $G$ is inner if and only if $d$ is inner [6]. For results concerning generalized derivations we refer the reader to $[1,6,14,16]$.

The purpose of this paper is to investigate generalized derivations $G$ on a complex Banach algebra $A$ such that the spectrum of $G x$ is finite for every $x \in A$. In particular, we will show that if $G$ is a generalized derivation defined on a complex semisimple Banach algebra $A$ such that $\sigma(G x)$ is finite for all $x \in A$, then $G A \subseteq \operatorname{soc} A$. Our results generalize those of $[7,8]$ which deal with derivations $d$ on a Banach algebra satisfying $\sharp \sigma(d x)<\infty$ for every $x \in A$. In $[4,5]$, one can find other conditions entailing that the range of a bounded derivation lies in the socle modulo the radical of a Banach algebra. It should be pointed out that inner generalized derivations $G$ defined on a Banach algebra $A$ such that $\sharp \sigma(G x)=1$ for every $x \in A$ were studied in [5].

Our study is closely connected with questions concerning derivations mapping into the radical. For details, we refer the readers to $[17,18]$, and

2000 Mathematics Subject Classification: 47B47, 47B48.

Key words and phrases: generalized derivations, finite spectrum, Banach algebras, socle. 
the references therein. We also mention the work of Curto and Mathieu [11], where spectrally bounded generalized inner derivations were investigated.

2. The case of dense algebras. We first give some tools and notation. Let $X$ be a vector space. As usual, $\mathcal{L}(X)$ denotes the algebra of all linear operators on $X$. If $X$ is a Banach space, the Banach algebra of all bounded linear operators on $X$ is denoted by $\mathcal{B}(X)$. The dual of $X$ will be denoted by $X^{*}$ and we will denote by $u \otimes f$ the linear operator on $X$ defined for any $u \in X$ and $f \in X^{*}$ by $(u \otimes f)(x)=f(x) u$ for $x \in X$. Moreover, $I$ denotes the identity mapping on $X$ and $\sharp F$ denotes the cardinality of a set $F$. Let $T$ be an operator on $X$. The point spectrum of $T$ is $\sigma_{\mathrm{p}}(T)=$ $\{\lambda \in \mathbb{C}: \lambda I-T$ is not injective $\}$.

Let $A$ be an arbitrary algebra. We denote by $\operatorname{rad} A$ the Jacobson radical of $A$, and by $\mathcal{Z}(A)$ the centre modulo the radical, defined by

$$
\mathcal{Z}(A)=\{a \in A: a x-x a \in \operatorname{rad} A \text { for all } x \in A\} .
$$

For every $a, b \in A$ let $\delta_{a, b}$ denote the inner generalized derivation defined by $\delta_{a, b}(x)=a x+x b$ for all $x \in A$. Recall that $\delta_{a}=\delta_{a,-a}$ is the inner derivation determined by $a$.

Now let $X$ be a Banach space and let $\mathcal{A}$ be a standard operator algebra on $X$. It is well-known that every derivation $d: \mathcal{A} \rightarrow \mathcal{A}$ is of the form $d S=T S-S T$ for some $T \in \mathcal{B}(X)[10]$. Similarly, we can prove the following result.

2.1. Lemma. Let $X$ be a complex Banach space and $\mathcal{A}$ a dense algebra of bounded linear operators on $X$. Suppose that $\mathcal{A}$ is closed and contains finite rank operators. Then every generalized derivation $G$ on $\mathcal{A}$ is of the form $G S=T S+S T^{\prime}$ for some $T, T^{\prime} \in \mathcal{B}(X)$.

Proof. Let $G$ be a generalized derivation on $\mathcal{A}$ determined by a derivation $d$. Since the algebra $\mathcal{A}$ is semisimple, $d$ and $G$ are continuous [15]. On the other hand, since $\mathcal{A}$ is a dense algebra containing finite rank operators, we check easily that $\mathcal{A}$ contains a rank one operator. Let $f$ be a nonzero linear functional such that $u \otimes f \in \mathcal{A}$ for some $0 \neq u \in X$. Applying again the density of $\mathcal{A}$, we see that $x \otimes f \in \mathcal{A}$ for all $x \in X$. Choose $v \in X$ such that $f(v)=1$ and define linear maps $T, T^{\prime}: X \rightarrow X$ by

$$
T x=(d(x \otimes f)) v, \quad T^{\prime} x=(G(x \otimes f)) v
$$

for all $x \in X$. We check at once that $T, T^{\prime}$ are continuous and $(d(S x \otimes f)) v=(d S) x+S(d(x \otimes f) v), \quad(G(S x \otimes f)) v=(G S) x+S(d(x \otimes f) v)$ for every $x \in \mathcal{A}$. As a result, $d S=T S-S T$ and $G S=T^{\prime} S-S T$ for all $S \in \mathcal{A}$. 
Our proofs involve techniques that have become standard in this area: the Jacobson density theorem, its generalizations and results on locally linearly dependent operators. Let $U$ and $V$ be vector spaces over a field $\mathbb{F}$ and let $V_{0}$ be a finite-dimensional subspace of $V$. Amitsur [2] proved that if $T_{1}, \ldots, T_{n}: U \rightarrow V$ are linear operators such that $T_{1} u, \ldots, T_{n} u$ are linearly dependent modulo $V_{0}$ for every $u \in U$, then there exist scalars $\alpha_{1}, \ldots, \alpha_{n}$, not all zero, such that $S=\alpha_{1} T_{1}+\cdots+\alpha_{n} T_{n}$ satisfies

$$
\operatorname{dim} S U \leq \operatorname{dim} V_{0}+\left(\begin{array}{c}
n+1 \\
2
\end{array}\right)-1
$$

Aupetit [3, p. 86] proved that if $U$ and $V$ are complex vector spaces and $V_{0}=\{0\}$, then $S$ can be chosen so that $\operatorname{rank} S \leq n-1$. Brešar and Šemrl [9, Theorem 2.2] extended Aupetit's result to the case of arbitrary infinite fields.

2.2. Theorem. Let $X$ be a complex vector space and $\mathcal{A}$ a dense algebra of linear operators on $X$. Suppose that there are linear operators $A, B$ on $X$ and an integer $n \in \mathbb{N}^{*}$ such that $\# \sigma_{\mathrm{p}}(A S+S B) \leq n$ for all $S \in \mathcal{A}$. Then there exist $\lambda \in \mathbb{C}$ and finite rank operators $F, F^{\prime} \in \mathcal{L}(X)$ such that $A=\lambda I+F$ and $B=-\lambda I+F^{\prime}$.

Proof. If $X$ is finite-dimensional, there is nothing to prove. So, assume that $X$ is infinite-dimensional. Suppose first that the set

$$
\left\{\xi_{1}, \ldots, \xi_{n+1}, B \xi_{1}, \ldots, B \xi_{n+1}\right\}
$$

is linearly independent for some $\xi_{1}, \ldots, \xi_{n+1}$ in $X$. Then there is $S \in \mathcal{A}$ such that $S \xi_{i}=0$ and $S B \xi_{i}=i \xi_{i}$. This entails that $(A S+S B) \xi_{i}=i \xi_{i}$ for each $1 \leq i \leq n+1$. Consequently, $\{1, \ldots, n+1\} \subseteq \sigma_{\mathrm{p}}(A S+S B)$, a contradiction. Thus for any $\xi_{1}, \ldots, \xi_{n+1}$ in $X$, the set $\left\{\xi_{1}, \ldots, \xi_{n+1}, B \xi_{1}, \ldots, B \xi_{n+1}\right\}$ is linearly dependent. According to [8, Lemma 3.1], there exists a finite rank operator $F^{\prime}$ such that $B=\lambda I+F^{\prime}$. Let $J$ be a basis of the subspace $F^{\prime} X$ and write $A=-\lambda I+F$ for some linear operator $F$.

We claim that $F$ has finite rank. Suppose this is not true and let $\xi_{1}, \ldots, \xi_{n+1} \in X$ be such that the set $\left\{F \xi_{1}, \ldots, F \xi_{n+1}\right\} \cup J$ is linearly independent. Then there exists $S \in \mathcal{A}$ such that $S J=\{0\}$ and $S F \xi_{i}=i \xi_{i}$ for each $1 \leq i \leq n+1$. This implies that $(A S+S B) F \xi_{i}=\left(F S+S F^{\prime}\right) F \xi_{i}=i F \xi_{i}$ and hence $\# \sigma_{\mathrm{p}}(A S+S B) \geq n+1$, a contradiction. Now the result follows from [2].

For a semisimple algebra $A$ the socle soc $A$ of $A$ is the sum of all minimal left ideals of $A$. If there are no minimal left ideals in $A$, then $\operatorname{soc} A=\{0\}$ by definition. The socle of $A$ is a direct sum of simple ideals. Now suppose that $A$ is a complex semisimple Banach algebra. Then every element of $\operatorname{soc} A$ has finite spectrum. Moreover, $\operatorname{soc} A$ is the largest algebraic ideal of $A$. 
2.3. Proposition. Let $X$ be a complex vector space and let $\mathcal{A}$ be a subalgebra of $\mathcal{L}(X)$ acting densely on $X$. Suppose that there are finite rank operators $F, F^{\prime}$ in $\mathcal{L}(X)$ satisfying $F S+S F^{\prime} \in \mathcal{A}$ for all $S \in \mathcal{A}$. Then $F^{\prime} \in \operatorname{soc} \mathcal{A}$ and $F \mathcal{A} \subseteq \mathcal{A}$.

Proof. If $X$ is finite-dimensional, we have $\mathcal{L}(X)=\mathcal{A}=\operatorname{soc} \mathcal{A}$. So suppose that $X$ is infinite-dimensional. Write $F=\sum_{i=1}^{p} u_{i} \otimes \varphi_{i}$ and $F^{\prime}=\sum_{j=1}^{r} v_{j} \otimes$ $\varphi_{j}^{\prime}$ for linearly independent sets $\left\{u_{1}, \ldots, u_{\mathrm{p}}\right\},\left\{v_{1}, \ldots, v_{r}\right\}$ of vectors in $X$ and linear functionals $\varphi_{1}, \ldots, \varphi_{\mathrm{p}}, \varphi_{1}^{\prime}, \ldots, \varphi_{r}^{\prime}$. Choose $w_{1}, \ldots, w_{r}$ in $X$ such that the set $\left\{w_{1}, \ldots, w_{r}, u_{1}, \ldots, u_{\mathrm{p}}\right\}$ is linearly independent. There are $S, S^{\prime} \in \mathcal{A}$ such that

$$
S v_{j}=w_{j}, \quad S^{\prime} w_{j}=v_{j}, \quad \text { and } \quad S^{\prime} u_{i}=0 \quad(1 \leq i \leq p, 1 \leq j \leq r) .
$$

Then

$$
S^{\prime}\left(F S+S F^{\prime}\right) \xi=F^{\prime} \xi
$$

for all $\xi \in X$. Hence $F^{\prime}=S^{\prime}\left(F S+S F^{\prime}\right) \in \mathcal{A}$. Finally, $F \mathcal{A} \subseteq \mathcal{A}$.

The above result is sharp in the following sense.

2.4. ExAmple. Let $X$ be a complex Banach space with a Schauder basis $\left\{e_{n}\right\}_{n=1}^{\infty}$ and suppose that the topological dual of $X$ is not separable (for instance, $X=l^{1}$ ). For every integer $n$, denote by $e_{n}^{*}$ the bounded linear functional on $X$ defined by $e_{n}^{*}\left(e_{m}\right)=\delta_{n}^{m}$ for every $m \in \mathbb{N}^{*}$. Let $\mathcal{A}$ be the closed subalgebra of $\mathcal{B}(X)$ generated by $u \otimes e_{n}^{*}$ for every integer $n$ and all $u \in X$. Observe that $\mathcal{A}$ is a dense algebra of linear operators on $X$. Let $f$ be a bounded linear functional on $X$ such that $f$ does not lie in the closed linear span of $\left\{e_{n}^{*}\right\}$. Pick $0 \neq u \in X$ and set $F=u \otimes f$. Then it is easy to check that $F \mathcal{A} \subseteq \mathcal{A}$, but $F \notin \mathcal{A}$.

2.5. Corollary. Let $X$ be a complex vector space and let $\mathcal{A}$ be a subalgebra of $\mathcal{L}(X)$ acting densely on $X$. Suppose that there are $A, B$ in $\mathcal{L}(X)$ satisfying $A S+S B \in \mathcal{A}$ for all $S \in \mathcal{A}$ and there exists $n \in \mathbb{N}^{*}$ such that $\# \sigma_{\mathrm{p}}(A S+S B) \leq n$ for all $S \in \mathcal{A}$. Then there exist finite rank operators $F, F^{\prime}$ in $\mathcal{L}(X)$ and a scalar $\lambda \in \mathbb{C}$ such that $F^{\prime} \in \operatorname{soc} \mathcal{A}, F \mathcal{A} \subseteq \mathcal{A}, A=\lambda I+F$ and $B=-\lambda I+F^{\prime}$.

Proof. According to Theorem 2.2, there exist finite rank operators $F, F^{\prime}$ $\in \mathcal{L}(X)$ satisfying $A=\lambda I+F$ and $B=-\lambda I+F^{\prime}$ for some scalar $\lambda \in \mathbb{C}$. Obviously, $F S+S F^{\prime} \in \mathcal{A}$ for all $S \in \mathcal{A}$. Now the above proposition yields the desired result.

3. The case of Banach algebras. We will denote the set of all primitive ideals in $A$ by $\operatorname{Prim}(A)$. Recall that primitive ideals are the kernels of irreducible representations of $A$. For every primitive ideal $P$ we denote by $\pi_{P}$ an irreducible representation of $A$ on a Banach space $X_{P}$ such that 
$\operatorname{Ker} \pi_{P}=P$. In particular, recall that the algebra $A / P$ can be seen as a subalgebra of $\mathcal{B}\left(X_{P}\right)$ acting densely on $X_{P}$. If $\operatorname{Prim}(A)$ is nonempty, we will often use the following result [19, Theorem 2.2.9]:

$$
\sigma(x) \cup\{0\}=\bigcup_{P \in \operatorname{Prim}(A)} \sigma(x+P) \cup\{0\} .
$$

Recall that for a given linear operator $T$ from a Banach space $X$ into a Banach space $Y$, the separating space of $T$ is the set $\mathcal{S}(T)=\left\{y \in Y\right.$ : there is a sequence $\left(x_{n}\right)_{n}$ in $X$ with $x_{n} \rightarrow 0$ and $\left.T x_{n} \rightarrow y\right\}$. Clearly, $\mathcal{S}(T)$ is a closed subspace of $Y$. By the closed graph theorem, $T$ is continuous if and only if $\mathcal{S}(T)=\{0\}$. Moreover, the map $\widehat{T}: X \rightarrow Y / \mathcal{S}(T)$ defined by $\widehat{T}(x)=T x+\mathcal{S}(T)$ is continuous. More details can be found in $[20]$.

3.1. Lemma. Let $A$ be a complex Banach algebra and let $G$ be a continuous generalized derivation on $A$ determined by a derivation $d$ of $A$. If $P$ is a primitive ideal of $A$, then $d P \subseteq P$.

Proof. Let $\left\{x_{k}\right\}$ be a sequence in $A$ such that $x_{k} \rightarrow 0$ and $d x_{k} \rightarrow y \in A$. Since $G$ is continuous, we infer that $0=\lim G\left(z x_{k}\right)=z y$ for each $z \in A$. Consequently, $A y=\{0\}$. Let us denote by $I$ the closed ideal

$$
I=\{u \in A: A u=\{0\}\} .
$$

Then $\mathcal{S}(d) \subseteq I \subseteq \operatorname{rad} A$. Consequently, the map $\bar{d}: A \rightarrow A / I$ defined by $\bar{d} a=d a+I$ is continuous. Next let $u \in I$. For all $x \in A$, we have

$$
0=d(x u)=x(d u) .
$$

It follows that $A(d u)=\{0\}$, which shows that $d I \subseteq I$. Now we can define the map $\widetilde{d}: A / I \rightarrow A / I$ such that $\widetilde{d}(a+I)=d a+I$. Note that $\widetilde{d}$ is continuous. According to [12, Proposition 2.7.22], $\widetilde{d}$ leaves invariant every primitive ideal of $A / I$. Let $P$ be a primitive ideal of $A$. Then $P / I$ is a primitive ideal of $A / I$. Thus, $d P \subseteq P+I=P$.

An algebra is said to be semiprime if $\{0\}$ is the only two-sided ideal $I$ for which $I^{2}=\{0\}$. Recall that every semisimple algebra is semiprime. Note the following consequence of the above proof.

3.2. Lemma. Let $A$ be a complex semiprime Banach algebra and let $G$ be a continuous generalized derivation on $A$ determined by a derivation $d$ of $A$. Then $d$ is continuous.

3.3. Remark. One is tempted to expect that the derivation $d$ in Lemma 3.1 is also continuous. But this is not true in general. Indeed, it follows from [18, Example 1.1] that there exists a Banach algebra $A$ and a discontinuous derivation $d$ on $A$ such that $A^{2} \neq\{0\}$ and $A(d A)=(d A) A=\{0\}$. 
Pick $a \in A$ such that $a A \neq\{0\}$. Let $G: A \rightarrow A$ be the left centralizer defined by $G x=a x$. Then $G$ is continuous. Moreover, $G$ can be seen as a generalized derivation determined by the derivation $d$.

We now come to our first general result.

3.4. Theorem. Let $A$ be a complex Banach algebra and let $G$ be a continuous generalized derivation on $A$ determined by a derivation $d$ of $A$. Suppose that $\sharp \sigma(G x)<\infty$ for all $x \in A$. Then there exists $a \in A$ such that $a+\operatorname{rad} A \in \operatorname{soc}(A / \operatorname{rad} A)$ and $d x-\delta_{a}(x) \in \operatorname{rad} A$ for all $x \in A$.

Proof. By [8, Lemma 2.1], there exists $n \in \mathbb{N}^{*}$ such that $\sharp \sigma(G x) \leq n$ for all $x \in A$. If $\operatorname{Prim}(A)$ is empty, there is nothing to prove. So suppose that $\operatorname{Prim}(A)$ is nonempty and let $P$ be a primitive ideal of $A$. By Lemma 3.1, $d P \subseteq P$, so denote by $d_{P}$ the induced derivation on $A / P$.

Our next step will be to prove that $d_{P}$ is of the form $d_{P}(S)=T S-$ $S T$ for some linear operator $T$ on $X_{P}$. Suppose that this is not true; then $X_{P}$ is infinite-dimensional. Let $\zeta_{1}, \ldots, \zeta_{n+1}$ be linearly independent vectors from $X_{P}$. Applying the Jacobson density theorem and [9, Theorem 3.6] we see that there exist $x, y \in A$ such that

$$
\left(\pi_{P} d(y)\right) \zeta_{i}=i \zeta_{i}, \quad\left(\pi_{P} y\right) \zeta_{i}=0, \quad\left(\pi_{P} x\right) \zeta_{i}=\zeta_{i} .
$$

This implies that $\left(\pi_{P} G(x y)\right) \zeta_{i}=i \zeta_{i}$. As a result, $\{1, \ldots, n+1\} \subseteq \sigma(G(x y))$, a contradiction.

Now let $T$ be a linear operator on $X_{P}$ such that $d_{P}(S)=T S-S T$ for every $S \in A / P$. Suppose that there are linearly independent vectors $\zeta_{1}, \ldots, \zeta_{n+1}$ in $X_{P}$ such that the set $\left\{\zeta_{1}, \ldots, \zeta_{n+1}, T \zeta_{1}, \ldots, T \zeta_{n+1}\right\}$ is linearly independent. Then we can choose $x, y \in A$ such that

$$
\left(\pi_{P}(y)\right) \zeta_{i}=0, \quad\left(\pi_{P}(y)\right)\left(T \zeta_{i}\right)=i \zeta_{i}, \quad\left(\pi_{P}(x)\right) \zeta_{i}=\zeta_{i} .
$$

Thus $\left(\pi_{P} G(x y)\right) \zeta_{i}=-i \zeta_{i}$ and $\{-1, \ldots,-(n+1)\} \subseteq \sigma(G(x y))$, a contradiction. It follows from [8, Lemma 3.1] that there exists $\lambda \in \mathbb{C}$ such that $T-\lambda I$ has finite rank. Clearly, $d_{P}(S)=\delta_{T-\lambda I}(S)$ for every $S \in A / P$. Thus, $\sigma(d x+P)$ is finite for all $x \in A$.

Now assume towards a contradiction that there exist distinct primitive ideals $P_{1}, \ldots, P_{n+1}$ of $A$ such that $d A \nsubseteq P_{i}$ for $1 \leq i \leq n+1$. For each $i \in\{1, \ldots, n+1\}$, let the inner derivation $d_{P_{i}}$ be implemented by the operator $T_{i}$. Then we can find $\zeta_{i} \in X_{P_{i}}$ such that the vectors $\zeta_{i}, T_{i} \zeta_{i}$ are linearly independent. Applying the extended Jacobson density theorem [13], we get elements $x, y \in A$ such that

$$
\pi_{i}(y) \zeta_{i}=0, \quad \pi_{i}(y) T_{i} \zeta_{i}=i \zeta_{i}, \quad \pi_{i}(x) \zeta_{i}=\zeta_{i}, \quad 1 \leq i \leq n+1 .
$$

This entails that $\left(\pi_{i} G(x y)\right) \zeta_{i}=-i \zeta_{i}$ for each $i$. Hence $\{-1, \ldots,-(n+1)\}$ $\subseteq \sigma(G(x y))$, a contradiction. 
We have thereby shown that $\sigma(d x)$ is finite for all $x \in A$. Using [ 5 , Theorem 2.4], we get the desired conclusion.

3.5. Proposition. Let $A$ be a complex Banach algebra and let $G$ be a continuous generalized derivation on A. Suppose that $\sharp \sigma(G x)<\infty$ for all $x \in A$. Then there exist at most a finite number of primitive ideals $P_{i}$ of $A$ such that $G(A) \nsubseteq P_{i}$.

Proof. Fix $n \in \mathbb{N}^{*}$ such that $\sharp \sigma(G x) \leq n$ for all $x \in A$. Suppose that $G$ is determined by a derivation $d$. It follows from Theorem 3.4 that there exists $a \in A$ such that $a+\operatorname{rad} A \in \operatorname{soc}(A / \operatorname{rad} A)$ and $d x-\delta_{a}(x) \in \operatorname{rad} A$ for all $x \in A$. Further, there exist at most a finite number of primitive ideals $P_{i}$ of $A$ such that $d A \nsubseteq P_{i}$.

Next assume that there exist distinct primitive ideals $P_{1}, \ldots, P_{n+1}$ of $A$ such that $d A \subseteq \bigcap_{i=1}^{n+1} P_{i}$ and $G A \nsubseteq P_{i}$ for $1 \leq i \leq n+1$. In order to simplify the notation we write $\pi_{i}, X_{i}$ instead of $\pi_{P_{i}}, X_{P_{i}}$ respectively. For $1 \leq i \leq n+1$, pick $x_{i} \in A$ such that $G x_{i} \notin P_{i}$ and choose $\zeta_{i} \in X_{i}$ such that $\left(\pi_{i}\left(G x_{i}\right)\right) \zeta_{i} \neq 0$. Applying the extended Jacobson density theorem [13], we can find $y_{i} \in A$ such that

$\pi_{i}\left(y_{i}\right)\left(\left(\pi_{i} G x_{i}\right) \zeta_{i}\right)=i \zeta_{i}, \quad \pi_{j}\left(y_{i}\right)\left(\left(\pi_{j} G x_{j}\right) \zeta_{j}\right)=0 \quad$ for $j \neq i, 1 \leq j \leq n+1$.

Set $x=x_{1} y_{1}+\cdots+x_{n+1} y_{n+1}$. Then $\pi_{i}(G x)\left(\left(\pi_{i} G x_{i}\right) \zeta_{i}\right)=i\left(\pi_{i} G x_{i}\right) \zeta_{i}$. We have proved that $\{1, \ldots, n+1\} \subseteq \sigma(G x)$. This contradiction completes the proof.

We are now in a position to prove our main result.

3.6. Theorem. Let $A$ be a complex Banach algebra and let $G$ be a continuous generalized derivation on A. Suppose that $\sharp \sigma(G x)<\infty$ for all $x \in A$. Then $G a+\operatorname{rad} A \in \operatorname{soc}(A / \operatorname{rad} A)$ for all $a \in A$. Moreover, if $A$ is unital then there are $u, v \in A$ such that $u+\operatorname{rad} A, v+\operatorname{rad} A \in \operatorname{soc}(A / \operatorname{rad} A)$ and $\left(G-\delta_{u, v}\right) A \subseteq \operatorname{rad} A$.

Proof. Fix $n \in \mathbb{N}^{*}$ such that $\sharp \sigma(G x) \leq n$ for every $x \in A$. Let $G$ be determined by the derivation $d$. It follows from Theorem 3.4 that there exists $a \in A$ such that $a+\operatorname{rad} A \in \operatorname{soc}(A / \operatorname{rad} A)$ and $d x-\delta_{a}(x) \in \operatorname{rad} A$ for all $x \in A$. Let $P$ be a primitive ideal of $A$. Since $\pi_{P}(a)$ has finite rank, there exists a finite-dimensional subspace $H$ of $X_{P}$ such that $X_{P}=\operatorname{Ker}\left(\pi_{P}(a)\right) \oplus H$.

Now assume towards a contradiction that there exists $x \in A$ such that $\pi_{P}(G x)$ has infinite rank. Then we check easily that there exist linearly independent vectors $\zeta_{1}, \ldots, \zeta_{n+1}$ in $\operatorname{Ker}\left(\pi_{P}(a)\right)$ such that the set $\left\{\zeta_{1}, \ldots, \zeta_{n+1}, \pi_{P}(G x) \zeta_{1}, \ldots, \pi_{P}(G x) \zeta_{n+1}\right\}$ is linearly independent and contained in $\operatorname{Ker} \pi_{P}(a)$. Now we can choose $y \in A$ such that

$$
\pi_{P}(y) \pi_{P}(G x) \zeta_{i}=i \zeta_{i}, \quad 1 \leq i \leq n+1 .
$$


This entails that

$$
\pi_{P}(G(x y))\left(\pi_{P}(G x)\right) \zeta_{i}=i\left(\pi_{P}(G x)\right) \zeta_{i}
$$

for each $i$. Consequently, $\{1, \ldots, n+1\} \subseteq \sigma(G(x y))$, a contradiction.

As a result, $G x+P \in \operatorname{soc}(A / P)$ for all $x \in A$. Now using the above proposition and [8, Proposition 2.2], we find that $G x+\operatorname{rad} A \in \operatorname{soc}(A / \operatorname{rad} A)$ for all $x \in A$.

Finally, suppose that $A$ is unital. Then

$$
G x=(G 1) x-\delta_{a}(x) \in \operatorname{rad} A, \quad \forall x \in A .
$$

3.7. Corollary. Let $A$ be a complex semisimple Banach algebra and let $G$ be a generalized derivation on $A$. Suppose that $\sharp \sigma(G x)<\infty$ for all $x \in A$. Then $G(A) \subseteq \operatorname{soc} A$. Moreover, if $A$ is unital then there exist $u, v \in \operatorname{soc} A$ such that $G=\delta_{u, v}$.

In the case of generalized inner derivations, we have the following characterization.

3.8. Theorem. Let $A$ be a complex Banach algebra and let $a, b \in A$. Then the following conditions are equivalent:

(1) $\# \sigma(a x+x b)<\infty$ for every $x$ in $A$,

(2) $a x+x b+\operatorname{rad} A \in \operatorname{soc}(A / \operatorname{rad} A)$ for every $x$ in $A$,

(3) there exist $u \in \mathcal{Z}(A)$ and $a^{\prime}, b^{\prime} \in A$ such that $a^{\prime}+\operatorname{rad} A, b^{\prime}+\operatorname{rad} A \in$ $\operatorname{soc}(A / \operatorname{rad} A)$ and $a=u+a^{\prime}, b=-u-b^{\prime}$.

Proof. The implication $(3) \Rightarrow(1)$ is clear, and $(1) \Rightarrow(2)$ is a direct consequence of Theorem 3.6. So suppose that (2) is true. Then $\sharp \sigma(a x+x b+$ $\operatorname{rad} A)<\infty$ for all $x \in A$. It follows from [3, Theorem 3.1.5] that $\sigma(a x+x b)$ is finite for all $x \in A$. Next we use the temporary notation $\bar{A}=A / \operatorname{rad} A$ and $x+\operatorname{rad} A=\bar{x}$ for every $x \in A$. Since the generalized derivation $\delta_{a, b}$ is determined by the inner derivation $\delta_{-b}$, Theorem 3.4 tells us that there exists $b^{\prime} \in A$ such that $\overline{b^{\prime}} \in \operatorname{soc} \bar{A}$ and $\delta_{b^{\prime}+b} A \subseteq \operatorname{rad} A$. Set $-u=b+b^{\prime}$. Then $u \in \mathcal{Z}(A)$ and $\left(\delta_{a, b}-\delta_{a-u,-b^{\prime}}\right) A \subseteq \operatorname{rad} A$. Applying again [3, Theorem 3.1.5], we infer that $\sharp \sigma\left(\delta_{a-u,-b^{\prime}} x\right)<\infty$ for all $x \in A$. By Theorem 3.6, $\overline{\delta_{a-u,-b^{\prime}}(A)} \subseteq \operatorname{soc} \bar{A}$. Since $\overline{b^{\prime}} \in \operatorname{soc} \bar{A}$, it follows that $(\overline{a-u}) \bar{A} \subseteq \operatorname{soc} \bar{A}$. Now it is easy to see that the ideal of $\bar{A}$ generated by $\overline{a-u}$ is algebraic. Consequently, $\overline{a-u} \in \operatorname{soc} \bar{A}$ and (3) is proved.

\section{References}

[1] E. Albas and N. Argac, Generalized derivations of prime rings, Algebra Colloq. 11 (2004), 399-410.

[2] S. A. Amitsur, Generalized polynomial identities and pivotal monomials, Trans. Amer. Math. Soc. 114 (1965), 210-226. 
[3] B. Aupetit, A Primer on Spectral Theory, Springer, New York, 1991.

[4] N. Boudi, Commutators with finite spectrum II, Acta Math. Sinica, to appear.

[5] N. Boudi and M. Mathieu, Commutators with finite spectrum, Illinois J. Math. 48 (2004), 687-699.

[6] M. Brešar, On the distance of the composition of two derivations to the generalized derivations, Glasgow Math. J. 33 (1991), 80-93.

[7] - Derivations mapping into the socle, II, Proc. Amer. Math. Soc. 126 (1998), 181-187.

[8] M. Brešar and P. Šemrl, Derivations mapping into the socle, Math. Proc. Cambridge Philos. Soc. 120 (1996), 339-346.

[9] - - - On locally linearly dependent operators and derivations, Trans. Amer. Math. Soc. 351 (1999), 1257-1275.

[10] P. R. Chernoff, Representations, automorphisms and derivations of some operator algebras, J. Funct. Anal. 12 (1973), 275-289.

[11] R. Curto and M. Mathieu, Spectrally bounded generalized inner derivations, Proc. Amer. Math. Soc. 123 (1995), 2431-2434.

[12] H. G. Dales, Banach Algebras and Automatic Continuity, London Math. Soc. Monogr. 24, Clarendon Press, Oxford 2000.

[13] J. M. G. Fell and R. S. Doran, Representations of *-Algebras, Locally Compact Groups, and Banach *-Algebraic Bundles, I, Academic Press, New York, 1988.

[14] B. Hvala, Generalized derivations in prime rings, Comm. Algebra 26 (1998), 11471166 .

[15] B. E. Johnson and A. M. Sinclair, Continuity of derivations and a problem of Kaplansky, Amer. J. Math. 90 (1968), 1067-1073.

[16] T. K. Lee, Generalized derivations of left faithful rings, Comm. Algebra 27 (1999), 4057-4073.

[17] M. Mathieu, Where to find the image of a derivation, in: Banach Center Publ. 30, Inst. Math., Polish Acad. Sci., 1994, 237-249.

[18] G. J. Murphy, Aspects of the theory of derivations, ibid., 207-211.

[19] C. E. Rickart, General Theory of Banach Algebras, Van Nostrand, 1960.

[20] A. M. Sinclair, Automatic Continuity of Linear Operators, London Math. Soc. Lecture Note Ser. 21, Cambridge Univ. Press, Cambridge, 1976.

Département de Mathématiques

Faculté des Sciences

Université My Ismail

Zitoune Meknes, Maroc

E-mail: nadia_boudi@yahoo.ca

ouchrif_said@hotmail.com

Received October 8, 2008

Revised version December 19, 2008

$(6426)$ 